\title{
Recent results of quarkonium and heavy flavour physics at ATLAS
}

\author{
Weimin Song ${ }^{1, *}$ \\ for the ATLAS Collaboration \\ ${ }^{1}$ Particle Physics Department, Rutherford Appleton Laboratory, Didcot, UK
}

\begin{abstract}
Heavy quark spectroscopy and exotic states are studied with the ATLAS detector, mainly through final states containing muon pairs from $J / \psi$ decays. This proceedings will summarise recent results from ATLAS on these topics, including production of quarkonium and heavy flavour, searches for exotic states and measurements of decay properties in open beauty production.
\end{abstract}

\section{Introduction}

Hardons have been used to test the particle physics Standard Model (SM) for a long time. The heavy flavour hadrons are massly produced in the proton proton collisions at Large Hadron Collider. With ATLAS detector, the production and decay of the heavy flavour hadrons could be studied. When comparing with the other detectors which are optimised for hadron physics study, such as Belle (II), BESIII, and LHCb, the advantage of ATLAS is that the number of recorded hardons is larger, while the disadvantage is the particle identification system is not designed for the separation between different hadrons. Normally, the $J / \psi \rightarrow \mu^{+} \mu^{-}$is required in the final state, in order to reduce the background, which is much more higher in the hadron collider when comparing with electron-positron collider.

In this proceedings, four recent publications from ATLAS are discussed, they are:

- Search for a Structure in the $B_{s}^{0} \pi^{ \pm}$Invariant Mass Spectrum with the ATLAS Experiment [1],

- Measurement of $b$-hadron pair production with the ATLAS detector in proton-proton collisions at $\sqrt{s}=8 \mathrm{TeV}[2]$,

- Measurement of quarkonium production in proton-lead and proton-proton collisions at 5.02 TeV with the ATLAS detector [3],

- Angular analysis of $B_{d}^{0} \rightarrow K^{*} \mu^{+} \mu^{-}$decays in $p p$ collisions at $\sqrt{s}=8 \mathrm{TeV}$ with the ATLAS detector [4].

\section{Is $X(5568)$ observed in the ATLAS dataset?}

In 2016, with $10.4 \mathrm{fb}^{-1}$ proton anti-proton collision data at center of mass energy of $1.96 \mathrm{TeV}$, the D0 collaboration made a claim about the discovery of $X(5568)$ in the $B_{s}^{0} \pi^{ \pm}$final states [5].

*e-mail: wesong@cern.ch 

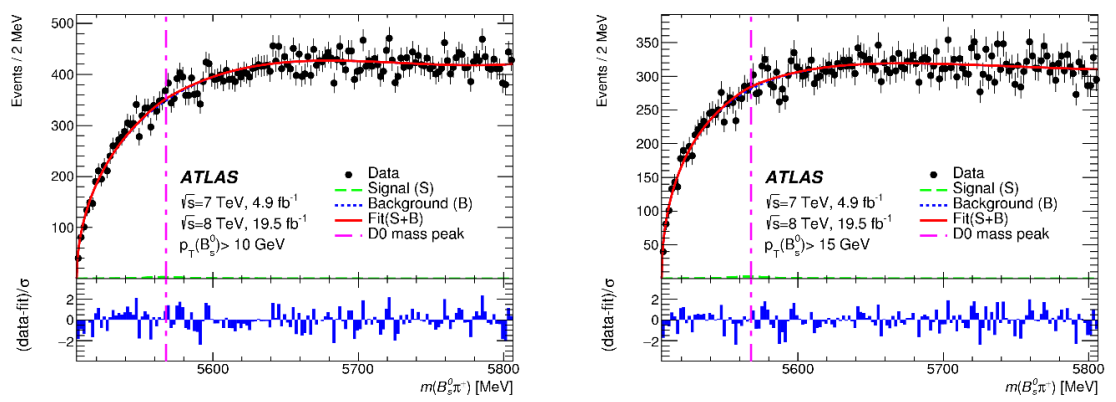

Figure 1: $B_{s}^{0} \pi^{ \pm}$mass distribution for candidates with $p_{T}\left(B_{s}^{0}\right)>10 \mathrm{GeV}$ (left) and $p_{T}\left(B_{s}^{0}\right)>15$ $\mathrm{GeV}$ (right). The bottom panels show the difference between each data point and the fit divided by the statistical uncertainty of that point.

The structure was interpreted as a tetraquark with four different quark flavors: $b, s, u$ and $d$. However, with similar method and same final state topology, neither CMS [6], LHCb [7] at LHC nor CDF [8] at Tevetran find any hint of $X(5568)$.

At ALTAS, the analysis is based on a data sample recorded with the ATLAS detector at the LHC corresponding to $4.9 \mathrm{fb}^{-1}$ of pp collisions at $7 \mathrm{TeV}$ and $19.5 \mathrm{fb}^{-1}$ at $8 \mathrm{TeV}$. No significant signal was found, as shown by Fig. 1. Upper limits on the number of signal events, with properties corresponding to those reported by $\mathrm{D} 0$, and on the $\mathrm{X}$ production rate relative to $B_{s}^{0}$ mesons, $\rho_{X}$, were determined at $95 \%$ confidence level. The results are $\mathrm{N}(\mathrm{X})<382$ and $\rho_{X}<0.016$ for $B_{s}^{0}$ mesons with transverse momenta above $10 \mathrm{GeV}$, and $\mathrm{N}(\mathrm{X})<356$ and $\rho_{X}<0.017$ for transverse momenta above $15 \mathrm{GeV}$.

The reason that the particle is observed by one experiment but not the other experiment could be physics also, rather than the mistakes made by experiments, as discussed by [9] [10]. More study is needed about the $X(5568)$ on both experimental and theoretical side, in order to pin down the nature of it.

\section{$3 b$-hadron pair production}

The production of heavy flavour quark in proton-proton collisions provides a fruitful testing ground for the predictions of quantum chromodynamics (QCD). There are a few methods that could be used to identify the heavy flavour quark, which will become a jet, in experiment. One popular method is based on the presence of a decay vertex or charged-particle tracks displaced from the primary interaction vertex due to the lifetime of the b-hadron, for example in [11]. The disadvantage of this method is that when the angle between two heavy flavour quarks is small, the performance of the tagging algorithm is poor due to the worse vertex fitting resolution.

ATLAS collaboration performed a new measurement of the production of two b-hadrons by tagging one b-hadron with $J / \psi(\rightarrow \mu \mu)+\mathrm{X}$ and the other with $\mu+\mathrm{Y}$, resulting in three muons in the final state. This means that the signature of the signal is: non-prompt $J / \psi$ decaying to two muons, and an third muon from the same interaction point. A number of normalised differential cross sections are also measured, and compared to predictions from the Pythia8, Herwig++, MadGraph5_aMC@NLO+Pythia8 and Sherpa event generators, providing constraints on heavy flavour production, as shown in Fig. 2. 

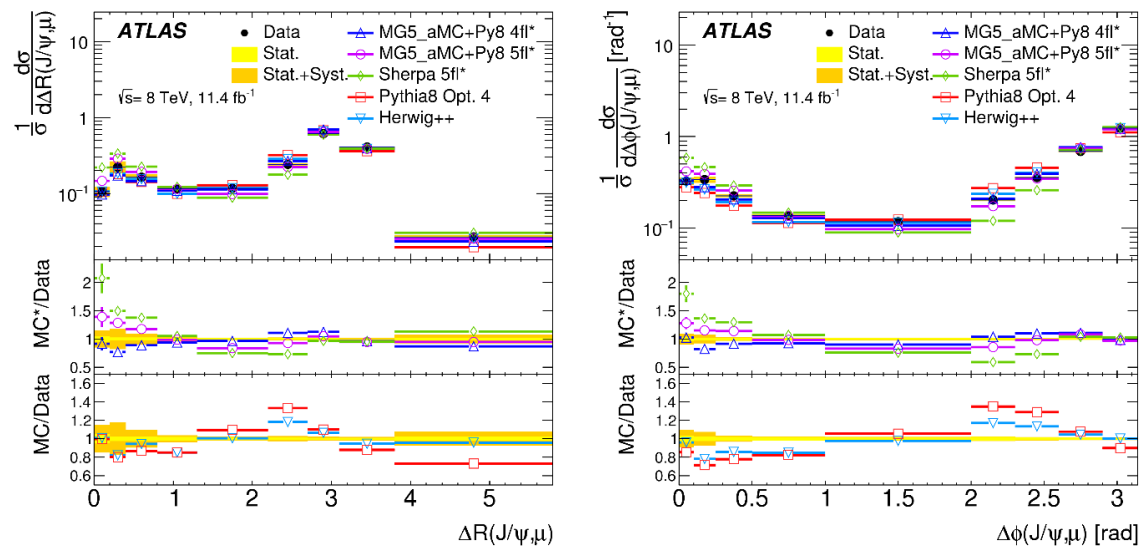

Figure 2: Measured normalised differential cross sections as a function of $\Delta R(J / \psi, \mu)$ $\Delta \phi(J / \psi, \mu)$.
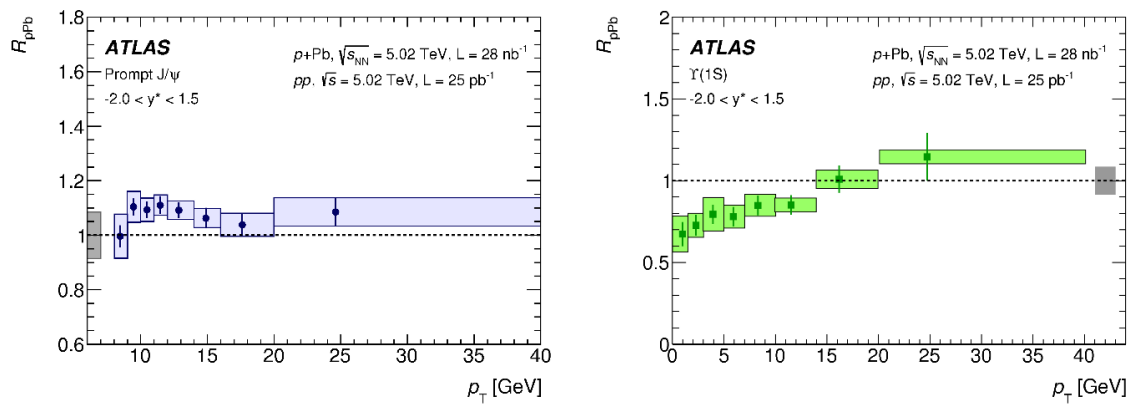

Figure 3: The nuclear modification factor, $\mathrm{RpPb}$, as a function of transverse momentum $\mathrm{pT}$ for prompt $J / \psi$ (left) and $\Upsilon(1 S)$.

\section{Quarkonium production}

The production of the quarkonium in the ultra-relativistic heavy-ion collisions will shed light on the mystery of the Quark-Gluon-Plasma. With integrated luminosities of $28 \mathrm{nb}^{-1} \mathrm{p}+\mathrm{pb}$ and $25 \mathrm{pb}^{-1}$ pp collision data, collected in 2013 and 2015 by the ATLAS detector at the LHC, both at a centre-of-mass energy per nucleon pair of $5.02 \mathrm{TeV}$, the production of charmonium and bottomnium are measured. No significant modification of the $J / \psi$ production is observed while $\Upsilon$ production is found to be suppressed at low transverse momentum in $\mathrm{p}+\mathrm{Pb}$ collisions relative to pp collisions, as shown in Fig. 3. The production of excited charmonium and bottomonium states is found to be suppressed relative to that of the ground states in central $\mathrm{p}+\mathrm{Pb}$ collisions.

\section{Angular analysis of $B_{d}^{0} \rightarrow K^{*} \mu^{+} \mu^{-}$decays}

Flavour-changing neutral current (FCNC) processes, such as $b \rightarrow s \mu^{+} \mu^{-}$, have played a significant role in the construction of the SM of particle physics. 

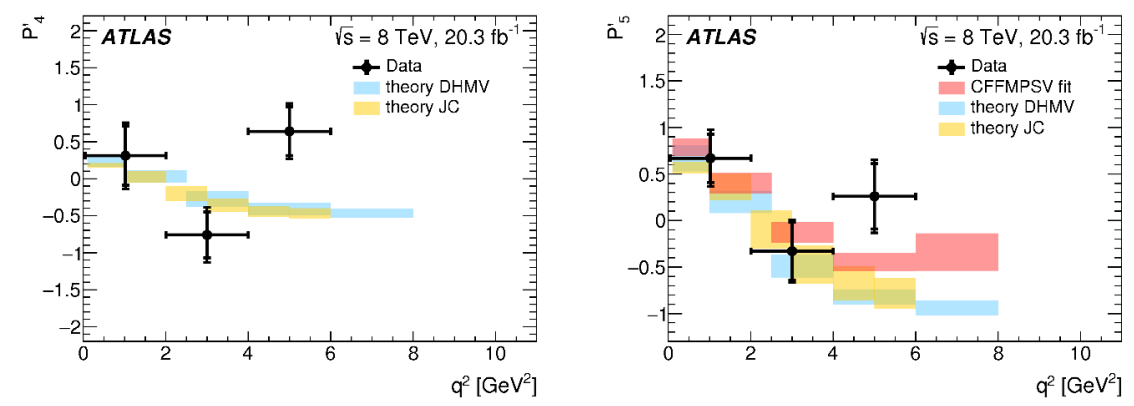

Figure 4: The measured values of $P_{4}^{\prime}$ (left) and $P_{5}^{\prime}$ (right) compared with predictions from the theoretical calculations. Statistical and total uncertainties are shown for the data, i.e. the inner mark indicates the statistical uncertainty and the total error bar the total uncertainty.

An angular analysis of the decay $B_{d}^{0} \rightarrow K^{*} \mu^{+} \mu^{-}$is published, based on proton-proton collision data recorded by the ATLAS experiment at the LHC. Measurements of the $K^{*}$ longitudinal polarisation fraction and a set of angular parameters obtained for this decay are presented. The results are compatible with the Standard Model predictions, with the exceptions for $P_{4}^{\prime}$ and $P_{5}^{\prime}$, as shown in Fig. 4.

\section{Summary}

The recent results about heavy flavour and quarkonium from ATLAS experiment are summarized here. These results help us step futher on the way of understanding the SM.

The speaker has received funding from the European Union's Horizon 2020 research and innovation programme under the Marie Skłodowska-Curie grant agreement (GA) No 665593 awarded to the Science and Technology Facilities Council.

\section{References}

[1] M. Aaboud et al. [ATLAS Collaboration], Phys. Rev. Lett. 120, no. 20, 202007 (2018)

[2] M. Aaboud et al. [ATLAS Collaboration], JHEP 1711, 062 (2017)

[3] M. Aaboud et al. [ATLAS Collaboration], Eur. Phys. J. C 78, no. 3, 171 (2018)

[4] M. Aaboud et al. [ATLAS Collaboration], arXiv:1805.04000 [hep-ex].

[5] V. M. Abazov et al. [D0 Collaboration], Phys. Rev. Lett. 117, no. 2, 022003 (2016)

[6] A. M. Sirunyan et al. [CMS Collaboration], Phys. Rev. Lett. 120, no. 20, 202005 (2018)

[7] R. Aaij et al. [LHCb Collaboration], Phys. Rev. Lett. 117, no. 15, 152003 (2016) Addendum: [Phys. Rev. Lett. 118, no. 10, 109904 (2017)]

[8] T. Aaltonen et al. [CDF Collaboration], Phys. Rev. Lett. 120, no. 20, 202006 (2018)

[9] Z. Yang, Q. Wang and U. G. Meißner, Phys. Lett. B 767, 470 (2017)

[10] H. W. Ke and X. Q. Li, arXiv:1802.08823 [hep-ph].

[11] M. Aaboud et al. [ATLAS Collaboration], Eur. Phys. J. C 76, no. 12, 670 (2016) 